\title{
Gravitational Field Equations on and off a 3-Brane World
}

\author{
A. N. Aliev \\ Feza Gürsey Institute, P.K. 6 Çengelköy, 81220 Istanbul, Turkey \\ A. E. Gümrükçüog̃lu \\ ITU, Faculty of Sciences and Letters, \\ Department of Physics, 34469 Maslak, Istanbul, Turkey
}

(Dated: August 20, 2018)

\begin{abstract}
The effective gravitational field equations on and off a 3 -brane world possessing a $Z_{2}$ mirror symmetry and embedded in a five-dimensional bulk spacetime with cosmological constant were derived by Shiromizu, Maeda and Sasaki (SMS) in the framework of the Gauss-Codazzi projective approach with the subsequent specialization to the Gaussian normal coordinates in the neighborhood of the brane. However, the Gaussian normal coordinates imply a very special slicing of spacetime and clearly, the consistent analysis of the brane dynamics would benefit from complete freedom in the slicing of spacetime, pushing the layer surfaces in the fifth dimension at any rates of evolution and in arbitrary positions. We generalize the SMS effective field equations on and off a 3-brane to the case where there is an arbitrary energy-momentum tensor in the bulk. We use a more general setting to allow for acceleration of the normals to the brane surface through the lapse function and the shift vector in the spirit of Arnowitt, Deser and Misner. We show that the gravitational influence of the bulk spacetime on the brane may be described by a traceless second-rank tensor $W_{i j}$, constructed from the "electric" part of the bulk Riemann tensor. We also present the evolution equations for the tensor $W_{i j}$, as well as for the corresponding "magnetic" part of the bulk curvature. These equations involve the terms determined by both the nonvanishing acceleration of normals in the nongeodesic slicing of spacetime and the presence of other fields in the bulk.
\end{abstract}




\section{INTRODUCTION}

The idea of a 3-brane world is strongly motivated by developments in string theory. String theory predicts the existence of higher-dimensional topological defects, D-branes, which are inherent in the theory and defined by the property that open string endpoints can live on them [1]. The attractive feature of the branes is that they have open string modes confined to them. In other words, open string modes which involve gauge fields, fermions and scalars of the Standard Model sector are trapped on the brane, while closed string modes of the gravity sector can propogate in higher dimensions. Another scenario of the localization of gauge fields arises in the framework of the Horava-Witten model [2]. In this model the strongly coupled ten-dimensional $E_{8} \times E_{8}$ heterotic string has been related to an eleven-dimensional theory (M-theory) compactified on an $S^{1} / Z_{2}$ orbifold with gauge fields propagating on two ten-dimensional branes located on the boundary hyperplanes with a $Z_{2}$ mirror symmetry. It has also been shown that there exists a subsequent compactification of this model on a deformed Calabi-Yau space, which leads to a five-dimensional spacetime with boundary hyperplanes becoming two 3-branes [3, 4]. The two 3-branes carry the matter and gauge fields on them and are identified with four-dimensional worlds. In this sense, one may phenomenologically think of a regime of five-dimensional bulk Universe which envelopes our physical four-dimensional Universe residing on a 3-brane.

Earlier, the idea that our physical Universe might reside on a $(3+1)$-dimensional hypersurface embedded in a higher dimensional space was suggested and studied in the framework of simple field-theoretical models not necessarily connected with string theory (see Refs.([5]-8]). However, a new striking feature of this idea has emerged in Arkani-HamedDimopoulos-Dvali (ADD) model [9], where by combining the 3-brane universe idea with the original idea of the Kaluza-Klein compactification, the authors suggested to solve the longstanding hierarchy problem of high-energy physics. The ADD model is built on the basic ingredients that our observable four-dimensional spacetime is a 3-brane with all Standard Model fields localized on it, while gravity is free to propagate in all extra spatial dimensions. The size of the extra dimensions may be much larger than the conventional Planckian length $\left(\sim 10^{-33} \mathrm{~cm}\right)$. Thus, in contrast to the original Kaluza-Klein scenario, in the ADD models the extra dimensions are supposed to manifest themselves as physical ones. One of the dramatic consequences of this model is that the fundamental scale of gravity might become 
as low as the ultraviolet scale of the Standard Model (of the order of a few TeV). This also raises the idea of $\mathrm{TeV}$-size mini black holes and their detectability in cosmic ray experiments or at future high energy colliders [10]-[12].

An alternative approach to solution of the hierarchy problem was put forward by Randall and Sundrum, who proposed a new higher-dimensional model consisting of a single extra spatial dimension [13]. This model (RS1) is based on two 3-branes that are located at the boundaries of an orbifold $S^{1} / Z_{2}$ in a five dimensional anti-de Sitter $\left(A d S_{5}\right)$ spacetime. Under a certain fine balance between the branes' self-gravity and bulk cosmological constant, the ultraviolet scale is generated from the large Planckian scale through an exponential warp function of a small compactification radius. Randall and Sundrum also proposed the second model (RS2) suggesting that our observable Universe resides on a single self-gravitating 3brane with positive tension, which is embedded in $A d S_{5}$ bulk spacetime with infinite extra dimension and negative cosmological constant [14]. In this model the fine tuning between the brane and the bulk leads to the phenomenon of localization on the 3-brane of a 5graviton zero mode along with rapid damping at large distances of massive KK modes. In other words, the RS2 model supports the conventional potential of Newtonian gravity on the 3-brane with high enough accuracy.

Due to their striking phenomenological consequences the ADD and RS braneworld models have caused enormous activity in particle physics, gravity and cosmology, paving the way to braneworld "scenarios" in these fields. A comprehensive description of these scenarios can be found in recent reviews [15]-[18]. Among them, the study of the properties of gravity localized around the branes is of particular interest. In [19]-[20] it has been shown that not only the ordinary Newtonian potential, but also four-dimensional Einstein gravity is localized around a 3-brane in RS2 braneworld, if in the linearized approach one properly takes into account the so-called brane-bending effect. A nonlinear model of plane wave propagating along the brane was studied in [21]. The subsequent analysis of this issue has been performed in [22]-[23], where the authors handled the problem in a nice covariant way, deriving the effective gravitational field equations on a 3-brane embedded in a five-dimensional bulk spacetime with $Z_{2}$-symmetry. These equations involve the higher-dimensional corrections to the ordinary Einstein equations, which are determined by a term quadratic in the energymomentum tensor of the brane, as well as a curvature term from the bulk space. These correction terms become ignorable in the low energy limit. Further generalizations of this 
approach to include the dilaton fields in the bulk, as well as high-order curvature terms of Gauss-Bonnet combinations have been given in [24]-[25]. The case of null radiation in the bulk was considered in [16].

The basic idea of the paper [22] and the other related works was to use the GaussCodazzi projective approach and project the field equations in the bulk on a 3-brane with the subsequent specialization to the Gaussian normal coordinates in the neighborhood of the 3-brane. However, as is well-known the Gaussian normal coordinates imply a very special slicing of spacetime in the sense that the geodesics orthogonal to a given hypersurface remain orthogonal to all successive hypersurfaces in the slicing [26]. This slicing may not affect the field equations on the brane, but it will change the off-brane evolution equations. Clearly, the consistent analysis of the brane dynamics would benefits from complete freedom in the slicing of spacetime by timelike hypersurfaces, pushing them in the fifth dimension at arbitrary rates and in arbitrary positions.

It is the purpose of this paper to present the effective gravitational field equations on a 3brane in the five-dimensional bulk, as well as the evolution equations off the brane in the case of arbitrary energy-momentum tensor in the bulk. We use, instead of the Gaussian normal coordinates, more general coordinate setting in the spirit of Arnowitt, Deser and Misner $(\mathrm{ADM})$ [27]. The use of the ADM type coordinates implies the slicing of the spacetime by timelike hypersurfaces pierced by a congruence of spacelike curves that are not geodesics and do not intersect the hypersurfaces orthogonally. In this approach one has freedom in analyzing the bulk dynamics in the most general case. We show that in the framework of this more general geometric setting the effective gravitational field equations on the 3-brane have the same form as obtained earlier [22] in the absence of matter fields in the bulk. We show that the gravitational influence of the bulk spacetime on the brane may be described through a traceless second-rank tensor $W_{i j}$, properly constructed from the "electric" part of the bulk Riemann tensor. We also show the evolution equations in the bulk are significantly modified due to the acceleration of normals in the nongeodesic slicing of spacetime and the presence of other fields in the bulk as well.

The paper is organized as follows. In Sec.II we describe the general formalism of a $(4+1)$ decomposition of a five-dimensional bulk spacetime using an approach similar to the ADM approach in the Hamiltonian formulation of four-dimensional general relativity. In Sec.III we begin with the decomposition of the connection coefficients using the usual definition of the 
Christoffel symbols. Then we derive the decomposition formulas for the Ricci, Einstein and Riemann tensors, in accordance with the $(4+1)$ splitting of the spacetime metric, without appealing to the Gauss-Codazzi projective approach. The effective field equations on the brane, as well as the conservation equations on it are given in Sec.IV. Finally, in Sec.V we derive the evolution equations off the brane for the corresponding projected parts of the curvature and energy-momentum tensors in the bulk space.

\section{GEOMETRIC SETTING}

We consider a five-dimensional bulk spacetime with manifold $M$ and metric $g_{A B}$ and suppose that the spacetime includes a 3-brane, a $(3+1)$-dimensional hypersurface, which, in turn, is endowed with the metric $h_{A B}$. Assuming that the bulk spacetime is covered by coordinates $x^{A}$ with $A=0,1,2,3,5$ we introduce an arbitrary scalar function

$$
Z=Z\left(x^{A}\right)
$$

such that $Z=$ const describes a layer surface in the family of non-intersecting timelike hypersurfaces $\Sigma_{Z}$, filling $M$, with the 3-brane located at the hypersurface $Z=0$.

The total action corresponding to the above setting consists of the Einstein-Hilbert action with bulk matter fields and the brane action

$$
S=\frac{1}{2 \kappa_{5}^{2}} \int_{M} d^{5} x \sqrt{-g}\left({ }^{(5)} R-2 \Lambda_{5}\right)+\int_{M} d^{5} x \sqrt{-g}^{(5)} L_{b u l k}+\int_{\text {brane }} d^{4} y \sqrt{-h} L_{\text {brane }},
$$

where $\kappa_{5}^{2}=8 \pi G_{5}$, with $G_{5}$ being the gravitational coupling constant, $y^{i}$ with $i=0,1,2,3$ are coordinates intrinsic to the brane and $\Lambda_{5}$ is the bulk cosmological constant. To avoid an undesired term in extremization of this action with respect to variations of $g_{A B}$ and $h_{A B}$, the Gibbons-Hawking boundary term [28] is also implied in it. The field equations derived from the action (2) have the form

$$
{ }^{(5)} G_{A B}={ }^{(5)} R_{A B}-\frac{1}{2} g_{A B}{ }^{(5)} R=-\Lambda_{5} g_{A B}+\kappa_{5}^{2}\left({ }^{(5)} T_{A B}+\sqrt{\frac{h}{g}} \tau_{A B} \delta(Z)\right),
$$

where ${ }^{(5)} T_{A B}$ is the energy-momentum tensor for the generic fields in the bulk, $\tau_{A B}$ is the energy-momentum tensor in the braneworld, the $\delta$-function singularity means that the brane energy-momentum has the form of a thin sheet distribution and the quantities $g$ and $h$ are the metric determinants of $g_{A B}$ and $h_{A B}$, respectively. 
The effective gravitational field equations in the braneworld are obtained by implementing a $(4+1)$ decomposition of the five-dimensional bulk spacetime, viewing it in terms of the four-dimensional hypersurface of a 3-brane and a spatial fifth dimension. (In the following we shall basically adopt the notations of Refs. [29]-[30] and use the reduced on-brane coordinate, instead of general five-dimensional ones). Clearly, one can introduce the unit spacelike normal to the brane surface as

$$
n_{A}=N \partial_{A} Z
$$

satisfying the normalization condition

$$
g_{A B} n^{A} n^{B}=1
$$

where the scalar function

$$
N=\left|g^{A B} \partial_{A} Z \partial_{B} Z\right|^{-1 / 2}
$$

is called the lapse function. The metric intrinsic to the brane can be obtained through defining the infinitesimal displacements within the brane. We recall the parametric equation of the brane $x^{A}=x^{A}\left(y^{i}\right)$, that implies the existence of a local frame given by the set of four vectors

$$
e_{i}^{A}=\frac{\partial x^{A}}{\partial y^{i}}
$$

which are tangent to the brane. It is clear that they satisfy the orthogonality condition

$$
n_{A} e_{i}^{A}=0
$$

With this local frame the infinitesimal displacements on the brane are determined by the induced metric

$$
h_{i j}=g_{A B} e_{i}^{A} e_{j}^{B}
$$

and its inverse, satisfying the completeness relation on the brane

$$
h^{i k} h_{k j}=\delta_{j}^{i}
$$

From equations (17) and (8) it follows that the bulk spacetime metric can be given as

$$
g_{A B}=n_{A} n_{B}+h_{i j} e_{A}^{i} e_{B}^{j}
$$

while the inverse metric has the form

$$
g^{A B}=n^{A} n^{B}+h^{i j} e_{i}^{A} e_{j}^{B} .
$$


In obtaining the last expression we have also used the completeness relation

$$
e_{A}^{i} e_{j}^{A}=\delta_{j}^{i}
$$

for the basis vectors on the brane.

Next, we shall introduce a spacelike vector $Z^{A}$, which formally can be thought of as an "evolution vector" into the fifth dimension. Having chosen the parameter along the orbits of this vector as $\mathrm{Z}$, we can write the relation

$$
Z^{A} \partial_{A} Z=1
$$

which means that the vector $Z^{A}$ is tangent to a congruence of curves intersecting the succesive hypersurfaces in the slicing of spacetime. We are interested in the general case, when the curves are not geodesics and they do not intersect the hypersurfaces orthogonally. The evolution vector $Z^{A}$ is not necessarily parallel to the normal vector $n^{A}$ and it can be decomposed into its normal and tangential parts

$$
Z^{A}=N n^{A}+N^{i} e_{i}^{A}
$$

where the four vector $N^{i}$ is known as the shift vector (see Ref.[30]). With this construction one can always define an alternative coordinate system $\left(y^{i}, y^{5}\right) \equiv\left(y^{i}, Z\right)$ on $M$, and express the spacetime metric in these new coordinates. Then the relations (6) and (13) imply that

$$
e_{i}^{A}=\left(\frac{\partial x^{A}}{\partial y^{i}}\right)_{Z}=\delta_{i}^{A}, \quad Z^{A}=\left(\frac{\partial x^{A}}{\partial Z}\right)_{y^{i}}=\delta_{Z}^{A}
$$

Furthermore, we note that

$$
d x^{A}=Z^{A} d Z+e_{i}^{A} d y^{i}=(N d Z) n^{A}+e_{i}^{A}\left(d y^{i}+N^{i} d Z\right)
$$

and the spacetime interval is decomposed into the form

$$
\begin{aligned}
d s^{2} & =g_{A B} d x^{A} d x^{B} \\
& =h_{i j} d y^{i} d y^{j}+2 N_{i} d y^{i} d Z+\left(N^{2}+N_{i} N^{i}\right) d Z^{2}
\end{aligned}
$$

It follows that the components of the five-dimensional metric can be written in terms of the induced 4-metric, the lapse and the shift functions as

$$
g_{A B}=\left(\begin{array}{cc}
h_{i j} & N_{i} \\
N_{j} & N^{2}+N_{i} N^{i}
\end{array}\right) .
$$


For the metric determinant we have

$$
\sqrt{-g}=N \sqrt{-h},
$$

while the inverse metric takes the form

$$
g^{A B}=\left(\begin{array}{cc}
h^{i j}+N^{i} N^{j} / N^{2} & -N^{i} / N^{2} \\
-N^{j} / N^{2} & 1 / N^{2}
\end{array}\right) .
$$

We also note that the covariant components of the spacelike unit normal vector in equation (41) are given by

$$
n_{A}=(0,0,0,0, N),
$$

and its contravariant components are obtained through the use of equations (5) and (18). We have

$$
n^{A}=\left(-\frac{N^{i}}{N}, \frac{1}{N}\right) .
$$

Finally, to describe the bending of the brane surface in the bulk we shall introduce the extrinsic curvature of the brane [30], calculating the derivative of the normal vector as

$$
\nabla_{A} n_{B}=K_{A B}+n_{A} a_{B}
$$

where $\nabla$ is the covariant derivative operator associated with the bulk metric $g_{A B}$, the extrinsic curvature tensor $K_{A B}$ is a symmetric tensor tangential to the brane $\left(K_{A B} n^{A}=0\right)$ and the 5 -acceleration of the normal vector is given by

$$
a_{A}=n^{B} \nabla_{B} n_{A}
$$

which with equations (15) and (22) can be written in the $(4+1)$ component form

$$
a_{A}=\left(a_{i}, a_{5}\right)=-\frac{1}{N}\left(D_{i} N, N^{i} D_{i} N\right) .
$$

Here $D$ is the covariant derivative operator defined with respect to the brane metric $h_{i j}$ and it is clear that $a_{A} n^{A}=0$.

The projected extrinsic curvature tensor is the symmetric 4-tensor given by

$$
K_{i j}=\nabla_{(B} n_{A)} e_{i}^{A} e_{j}^{B}=\frac{1}{2}\left(£_{n} g_{A B}\right) e_{i}^{A} e_{j}^{B},
$$


where the symbol $£$ stands for the Lie derivative. The extrinsic curvature can be related to the evolution of the brane metric into the fifth spatial dimension through its Lie derivative along the evolution vector $Z^{A}$. We obtain that

$$
K_{i j}=\frac{1}{2 N}\left[\left(£_{Z} g_{A B}\right) e_{i}^{A} e_{j}^{B}-D_{i} N_{j}-D_{j} N_{i}\right],
$$

which, with equations in (15), reduces to the form

$$
K_{i j}=\frac{1}{2 N}\left(\partial_{5} h_{i j}-D_{i} N_{j}-D_{j} N_{i}\right) .
$$

Here and in what follows $\partial_{5}=\partial / \partial Z$ and the indices of all 4 -tensors intrinsic to the brane are raised and lowered with respect to the metric $h_{i j}$.

\section{DECOMPOSITION OF CONNECTION COEFFICIENTS AND CURVATURE TENSORS}

In accordance with a $(4+1)$ decomposition of the five dimensional spacetime metric given in (177), we now need to establish the reduction formulas between the five dimensional connection coefficients and curvature tensors and the quantities characterizing the intrinsic and extrinsic properties of the brane surface. We start with the usual definition of the Christoffel symbols in five dimensions

$$
\Gamma_{B C}^{A}=\frac{1}{2} g^{A D}\left(\partial_{B} g_{C D}+\partial_{C} g_{B D}-\partial_{D} g_{B C}\right),
$$

and substituting into it the metric decompositions given in (18) and (201), we obtain that the nonvanishing components of the Christoffel symbols are split into the form

$$
\begin{aligned}
& \Gamma_{i j}^{5}=-\frac{1}{N} K_{i j}, \\
& \Gamma_{i 5}^{5}=N^{j} \Gamma_{i j}^{5}+\frac{1}{N} D_{i} N, \\
& \Gamma_{j l}^{i}=\lambda_{j l}^{i}-N^{i} \Gamma_{j l}^{5}, \\
& \Gamma_{j 5}^{i}=-N^{i} \Gamma_{5 j}^{5}+N K_{j}^{i}+D_{j} N^{i}, \\
& \Gamma_{55}^{5}=N^{i} \Gamma_{i 5}^{5}+\frac{1}{N} \partial_{5} N, \\
& \Gamma_{55}^{i}=-N^{i} \Gamma_{55}^{5}+\partial_{5} N^{i}+N^{j} D_{j} N^{i}+N\left(2 K_{j}^{i} N^{j}-D^{i} N\right),
\end{aligned}
$$

where the quantities

$$
\lambda_{j l}^{i}=\frac{1}{2} h^{i m}\left(\partial_{j} h_{l m}+\partial_{l} h_{j m}-\partial_{m} h_{j l}\right)
$$


are the Christoffel symbols on the brane formed from the metric $h_{i j}$.

The decomposition of the five dimensional Ricci tensor can be obtained through the use of the above reduction formulas (30) and (19) in the general expression

$$
{ }^{(5)} R_{A B}=\partial_{C} \Gamma_{A B}^{C}-\partial_{A} \partial_{B}(\ln \sqrt{-g})+\Gamma_{A B}^{C} \partial_{C}(\ln \sqrt{-g})-\Gamma_{A D}^{C} \Gamma_{B C}^{D} .
$$

After performing straightforward calculations we find that

$$
\begin{aligned}
{ }^{(5)} R_{i j}= & R_{i j}-\frac{1}{N}\left[\left(\partial_{5}-£_{\vec{N}}\right) K_{i j}+D_{i} D_{j} N\right]+2 K_{i}^{m} K_{m j}-K K_{i j}, \\
{ }^{(5)} R_{5 i}= & { }^{(5)} R_{i j} N^{j}-N\left(D_{i} K-D_{j} K_{i}^{j}\right), \\
{ }^{(5)} R_{55}= & { }^{(5)} R_{i j} N^{i} N^{j}-N\left(\partial_{5} K+\square N\right)-N^{2} K_{l m} K^{l m} \\
& +2 N N^{j} D_{i}\left(K_{j}^{i}-\frac{1}{2} \delta_{j}^{i} K\right),
\end{aligned}
$$

where $R_{i j}$ is the Ricci curvature of the braneworld, $K=h^{i j} K_{i j}$ is the trace of the extrinsic curvature, the operator $\square=D_{m} D^{m}$ is the D'Alembertian acting on the brane and the Lie derivative $£_{\vec{N}}$ is taken along the shift vector $N^{i}$.

Similarily, for the five dimensional Ricci scalar we obtain the following reduction formula

$$
{ }^{(5)} R=R-\frac{2}{N}\left(\partial_{5} K-£_{\vec{N}} K+\square N\right)-K_{l m} K^{l m}-K^{2},
$$

with the four-dimensional Ricci scalar $R$ defined on the brane. We note that in obtaining the above expressions we have repeatedly used the following useful relations

$$
\begin{aligned}
\partial_{5} h^{i j} & =-h^{i m} h^{j l} \partial_{5} h_{l m}, \\
\partial_{5} \ln \sqrt{-h} & =N K+D_{i} N^{i}, \\
h^{i j} \partial_{5} K_{i j} & =\partial_{5} K+2\left(K_{j}^{i} D_{i} N^{j}+N K_{i j} K^{i j}\right) .
\end{aligned}
$$

We can now establish the reduction formulas for the components of the Einstein tensor in equation (33). Using equations given in (33), (34) and taking into account relations (35) we obtain

$$
\begin{aligned}
&{ }^{(5)} G_{i j}= G_{i j}-\frac{1}{N}\left[\left(\partial_{5}-£_{\vec{N}}\right)\left(K_{i j}-h_{i j} K\right)+D_{i} D_{j} N\right]-3 K K_{i j}+2 K_{i}^{m} K_{m j} \\
&+\frac{1}{2} h_{i j}\left(K^{2}+K_{l m} K^{l m}+\frac{2}{N} \square N\right), \\
&{ }^{(5)} G_{i 5}={ }^{(5)} G_{i j} N^{j}-N\left(D_{i} K-D_{j} K_{i}^{j}\right), \\
&{ }^{\left.{ }^{5}\right)} G_{55}={ }^{(5)} G_{i j} N^{i} N^{j}-\frac{1}{2} N^{2}\left(R-K^{2}+K_{l m} K^{l m}\right)+2 N N^{j} D_{i}\left(K_{j}^{i}-\delta_{j}^{i} K\right),
\end{aligned}
$$


where $G_{i j}$ is the Einstein 4-tensor in the braneworld.

We shall also need the decomposition formulas for the Riemann curvature tensor of the metric (17). They can be obtained from the general expression

$$
{ }^{(5)} R_{B C D}^{A}=\partial_{C} \Gamma_{B D}^{A}-\partial_{D} \Gamma_{B C}^{A}+\Gamma_{B D}^{M} \Gamma_{C M}^{A}-\Gamma_{B C}^{M} \Gamma_{D M}^{A}
$$

by substituting into it the decomposition of the Christoffel symbols given in equation (30). Having done this, for the covariant components of the five dimensional Riemann curvature we find

$$
\begin{aligned}
{ }^{(5)} R_{i j l m}= & R_{i j l m}+K_{i m} K_{j l}-K_{i l} K_{j m}, \\
{ }^{(5)} R_{5 i j l}= & { }^{(5)} R_{m i j l} N^{m}-N\left(D_{j} K_{i l}-D_{l} K_{i j}\right), \\
{ }^{(5)} R_{5 i 5 j}= & { }^{(5)} R_{5 i m j} N^{m}-N\left[\left(\partial_{5}-£_{\vec{N}}\right) K_{i j}+D_{i} D_{j} N\right] \\
& +N\left[N^{m}\left(D_{i} K_{m j}-D_{m} K_{i j}\right)+N K_{i}^{m} K_{m j}\right],
\end{aligned}
$$

where the unmarked Riemann tensor is intrinsic to the brane surface. In the derivation of these expressions, in addition to equations in (35), we have also used the relation

$$
\partial_{5} \lambda_{j l}^{i}=R_{l m j}^{i} N^{m}+D_{j} D_{l} N^{i}+D_{j}\left(N K_{l}^{i}\right)+D_{l}\left(N K_{j}^{i}\right)-D^{i}\left(N K_{j l}\right)
$$

It is important to note that among equations in (38) only the last equation involves the term describing the evolution into the fifth dimension. We recall that the first equation is known as the Gauss equation. In the case of vanishing acceleration of the normals $\left(N=1, N^{i}=0\right)$, the above formulas are in agreement with those obtained in 25] within the Gauss-Codazzi projective approach.

\section{EFFECTIVE EQUATIONS ON THE BRANE}

We are now in position to write down the effective gravitational field equations on a 3-brane substituting the reduction formulas (36) into the basic equation (3) together with a suitable decomposition of its right-hand-side. First, we note that

$$
\begin{aligned}
{ }^{(5)} G_{i 5} & ={ }^{(5)} G_{i A} Z^{A}={ }^{(5)} G_{i j} N^{j}+N{ }^{(5)} G_{i A} n^{A}, \\
{ }^{(5)} G_{55} & ={ }^{(5)} G_{A B} Z^{A} Z^{B} \\
& ={ }^{(5)} G_{i j} N^{i} N^{j}+2 N{ }^{(5)} G_{i A} N^{i} n^{A}+N^{2}{ }^{(5)} G_{A B} n^{A} n^{B}
\end{aligned}
$$


and comparing these expressions with the second and third equations in (36) we arrive at equations

$$
\begin{gathered}
\frac{1}{2}\left(R-K^{2}+K_{l m} K^{l m}\right)=\Lambda_{5}-\kappa_{5}^{2} P \\
D_{m} K_{i}^{m}-D_{i} K=\kappa_{5}^{2} J_{i}
\end{gathered}
$$

where $P={ }^{(5)} T_{A B} n^{A} n^{B}$ is a sort of "pressure" exerted on the brane, while the 4-vector $J_{i}={ }^{(5)} T_{i B} n^{B}$ describes the energy-momentum flux onto or from the brane. Using the analogy with the Hamiltonian formulation of general relativity [27] we can think of these equations as the Hamiltonian constraint and the momentum constraint equations on the brane, respectively.

Next, we insert the remaining first equation in (36) into equation (3) and rewrite it in form

$$
\begin{aligned}
G_{i j} & -\frac{1}{N}\left[\left(\partial_{5}-£_{\vec{N}}\right)\left(K_{i j}-h_{i j} K\right)+D_{i} D_{j} N\right]-3 K K_{i j}+2 K_{i}^{m} K_{m j} \\
& +\frac{1}{2} h_{i j}\left(K^{2}+K_{l m} K^{l m}+\frac{2}{N} \square N\right)=-\Lambda_{5} h_{i j}+\kappa_{5}^{2}\left({ }^{(5)} T_{i j}+\sqrt{\frac{h}{g}} \tau_{i j} \delta(Z)\right) .
\end{aligned}
$$

In order to assign to this equation a physical meaning on the brane we must do two things. First, we must relate the $\delta$-function behaviour in the brane energy-momentum tensor on the right-hand-side of equation (44) to the jump in the extrinsic curvature of the brane on its evolution into the fifth dimension. Further, we must express the term describing the evolution into the extra dimension in terms of four-dimensional quantities on the brane, more precisely in terms of their limiting values on the brane. At this stage, it is useful to impose on the brane $Z_{2}$-symmetry and integrate with an appropriate weight the equation (44) across the brane surface along the orbits of the evolution vector $Z^{A}$. Then passing to the zero limit $Z \rightarrow \pm 0$ and assuming that the quantities $K_{i j}^{+}$and $K_{i j}^{-}$evaluated on both sides of the brane, respectively, remain bounded, we arrive at Israel's junction condition [29]

$$
\begin{aligned}
& {\left[h_{i j}\right]_{ \pm}=h_{i j}^{+}-h_{i j}^{-}=0} \\
& {\left[K_{i j}\right]_{ \pm}-h_{i j}[K]_{ \pm}=-\kappa_{5}^{2} \tau_{i j} .}
\end{aligned}
$$

Since we have imposed on the brane $Z_{2}$-symmetry, that implies $Z^{A} \rightarrow-Z^{A} \quad\left(n^{A} \rightarrow-n^{A}\right)$ and hence,

$$
K_{i j}^{+}=-K_{i j}^{-}
$$


Taking this into account in equation (45) and omitting the indices \pm we obtain

$$
K_{i j}=-\frac{1}{2} \kappa_{5}^{2}\left(\tau_{i j}-\frac{1}{3} h_{i j} \tau\right)
$$

where $\tau=\tau_{i j} h^{i j}$ is the trace of the energy-momentum tensor of the brane.

Next, we note that

$$
{ }^{(5)} R_{5 i 5 j}={ }^{(5)} R_{A B C D} Z^{A} Z^{C} e_{i}^{B} e_{j}^{D},
$$

which by means of equation (14) and the second equation in (38) can be written in the form

$$
{ }^{(5)} R_{5 i 5 j}=N^{2} A_{i j}+{ }^{(5)} R_{5 i m j} N^{m}-N B_{j i m} N^{m},
$$

where we have introduced the quantities

$$
\begin{aligned}
A_{i j} & ={ }^{(5)} R_{A B C D} n^{A} n^{C} e_{i}^{B} e_{j}^{D}, \\
B_{j i m} & ={ }^{(5)} R_{A B C D} n^{A} e_{j}^{B} e_{i}^{C} e_{m}^{D}=2 D_{[m} K_{i] j},
\end{aligned}
$$

which, by analogy with their corresponding counterparts in ordinary general relativity [31], can be thought of as the "electric" part and the "magnetic" part of the bulk Riemann tensor, respectively. Comparing equation (49) with the last equation in (38) we find that

$$
-\frac{1}{N}\left(\partial_{5} K_{i j}-£_{\vec{N}} K_{i j}\right)=A_{i j}-K_{i}^{m} K_{m j}-D_{i} a_{j}+a_{i} a_{j}
$$

where we have inserted the four-dimensional acceleration $a_{i}=a_{A} e_{i}^{A}$ given in (25). Similarily, from the reduction formula (34) for the Ricci scalar, it follows that

$$
\frac{1}{N}\left(\partial_{5}-£_{\vec{N}}\right) K=\frac{1}{2}\left(R-{ }^{(5)} R-K_{l m} K^{l m}-K^{2}\right)+D_{m} a^{m}-a^{2},
$$

where $a^{2}=a_{m} a^{m}$. Taking into account the obvious relation

$$
\frac{1}{N}\left(\partial_{5}-£_{\vec{N}}\right) K h_{i j}=\frac{1}{N} h_{i j}\left(\partial_{5} K-£_{\vec{N}} K\right)+2 K K_{i j}
$$

and combining equations (51) and (52) we arrive at desired expression relating the evolution term in (44) to the four-dimensional quantities on the brane

$$
\begin{aligned}
-\frac{1}{N}\left(\partial_{5}-£_{\vec{N}}\right)\left(K_{i j}-h_{i j} K\right)= & A_{i j}+\frac{1}{2} h_{i j}\left(R-K_{l m} K^{l m}-K^{2}-{ }^{(5)} R\right)-K_{i}^{m} K_{m j} \\
& +2 K K_{i j}-D_{i} a_{j}+a_{i} a_{j}+h_{i j}\left(D_{m} a^{m}-a^{2}\right) .
\end{aligned}
$$


Inserting this expression into (44) and approaching the brane from either + or - side, we obtain the effective gravitational field equations on the brane in the form

$$
\begin{aligned}
G_{i j} & +\frac{1}{2} h_{i j}\left(K^{2}-K_{l m} K^{l m}\right)+K_{i}^{m} K_{m j}-K K_{i j}+A_{i j} \\
& =-\frac{1}{3} h_{i j} \Lambda_{5}+\kappa_{5}^{2}\left[{ }^{(5)} T_{i j}+h_{i j}\left(P-\frac{1}{3}{ }^{(5)} T\right)\right]
\end{aligned}
$$

where we have used equation (42), while the extrinsic curvature terms are determined through equation (47).

It may be useful to introduce a traceless tensor $W_{i j}$, constructed from the electric part of the Riemann curvature according to the relation

$$
W_{i j}=A_{i j}-\frac{1}{4} h_{i j} A
$$

where $A$ is the trace of $A_{i j}$ with respect to the induced metric $h_{i j}$. We have

$$
A=\frac{1}{2}\left({ }^{(5)} R-R-K_{l m} K^{l m}+K^{2}\right) .
$$

Substituting this new tensor into equation (55) and expressing the extrinsic curvature terms through the energy-momentum tensor in the braneworld, we obtain the alternative form of the effective field equations

$$
G_{i j}=-\frac{1}{2} h_{i j}\left(\Lambda_{5}-\kappa_{5}^{2} P\right)-W_{i j}-3 \kappa_{5}^{2} U_{i j}-\kappa_{5}^{4} \tilde{T}_{i j}
$$

where the source term $\tilde{T}_{i j}$ is quadratic in the energy-momentum tensor of the brane and given by

$$
\tilde{T}_{i j}=\frac{1}{4}\left[\tau_{i}^{m} \tau_{m j}-\frac{1}{3} \tau \tau_{i j}-\frac{1}{2} h_{i j}\left(\tau_{l m} \tau^{l m}-\frac{1}{3} \tau^{2}\right)\right]
$$

while

$$
U_{i j}=-\frac{1}{3}\left({ }^{(5)} T_{i j}-\frac{1}{4} h_{i j} h^{l m(5)} T_{l m}\right),
$$

is the traceless, with respect to the metric $h_{i j}$, projection of the bulk energy-momentum tensor onto the brane. On the other hand, taking into account the decomposition of the Riemann tensor in five dimensions in terms of the traceless Weyl tensor $C_{A B C D}$ and the Ricci tensor

$$
R_{A B C D}=C_{A B C D}+\frac{2}{3}\left(g_{A[C} R_{D] B}-g_{B[C} R_{D] A}\right)-\frac{1}{6} R g_{A[C} g_{D] B}
$$


and passing from the electric part of the Riemann tensor $A_{i j}$ in equation (50) to that of the Weyl tensor defined as

$$
E_{i j}={ }^{(5)} C_{A B C D} n^{A} n^{C} e_{i}^{B} e_{j}^{D}
$$

we find that

$$
A_{i j}=E_{i j}+\frac{1}{3}\left[{ }^{(5)} R_{i j}+\frac{1}{4} h_{i j}{ }^{(5)} R-\frac{1}{2} h_{i j}\left(R-K^{2}+K_{l m} K^{l m}\right)\right] .
$$

Using this equation one can easily relate the tensors $W_{i j}$ and $E_{i j}$. We find that

$$
E_{i j}=W_{i j}+\kappa_{5}^{2} U_{i j}
$$

which shows that the traceless projection of the bulk energy-momentum tensor (60) determines the difference between the electric part of the five-dimensional Weyl tensor and the traceless tensor $W_{i j}$, constructed from the electric part of the Riemann tensor as in equation (56). In the particular case, where the energy-momentum tensor of the braneworld admits the form [22]

$$
\tau_{i j}=-\lambda h_{i j}+S_{i j}
$$

where $\lambda$ is the brane tension, we can transform equation (58) into the following form

$$
G_{i j}=-\Lambda h_{i j}+\kappa_{4}^{2} S_{i j}+\kappa_{5}^{4} M_{i j}-W_{i j}-3 \kappa_{5}^{2} U_{i j}
$$

where

$$
\begin{aligned}
\Lambda & =\frac{1}{2}\left(\Lambda_{5}+\frac{1}{6} \kappa_{5}^{4} \lambda^{2}-\kappa_{5}^{2} P\right), \\
\kappa_{4}^{2} & =\frac{1}{6} \kappa_{5}^{4} \lambda \\
M_{i j} & =-\frac{1}{4}\left[\left(S_{i}^{m} S_{m j}-\frac{1}{3} S S_{i j}\right)-\frac{1}{2} h_{i j}\left(S_{l m} S^{l m}-\frac{1}{3} S^{2}\right)\right] .
\end{aligned}
$$

It is important to note that these equations do not involve any terms determined by a nonvanishing accerelation of normals inherent in the ADM type foliation of spacetime. In the absence of the energy-momentum sources in the bulk, equations (66) have exactly the same form as the effective field equations derived by Shiromizu, Maeda and Sasaki (SMS) in [22]. Thus, the form of the SMS equations still continues to be held in the ADM type non-geodesic slicing of a spacetime manifold.

We see that the effective field equations (66) drastically differ from the usual fourdimensional Einstein equations not only due to the term quadratic in the energy-momentum 
tensor of the brane (69), but also due to the curvature and energy-momentum terms from the bulk space. In other words, the gravitational effects of the bulk are transmitted to the brane through a traceless second-rank tensor $W_{i j}$, while the influence of the bulk space energy and momentum on the brane is felt through both the normal compressive pressure and the traceless in-brane components of the bulk energy-momentum tensor. On these grounds, it is clear that the effective equations on the brane are not closed, thereby there exists a continuous exchange of energy and momentum between the brane and the bulk.

In order to demonstrate these features we appeal to equation (43) and insert into it the relation given in (47). Then we arrive at the following equation of "continuity"

$$
D_{m} \tau_{i}^{m}=-2^{(5)} J_{i}
$$

which manifests the existence of an energy flux onto, or away from the brane. In the case where ${ }^{(5)} J_{i} \equiv 0$, we have conservation equation for energy-momentum on the brane.

Next, we calculate the divergence of equation (158). Taking into account the contracted Bianchi identities

$$
D^{i} G_{i j}=0
$$

after some rearrangements we find that

$$
\begin{aligned}
D^{i}\left(W_{i j}+3 \kappa_{5}^{2} U_{i j}-\frac{1}{2} \kappa_{5}^{2} h_{i j} P\right)= & \frac{1}{4} \kappa_{5}^{4}\left[\tau^{m i}\left(D_{j} \tau_{m i}-D_{i} \tau_{m j}\right)+\frac{1}{3}\left(\tau_{j}^{i}-\delta_{j}^{i} \tau\right) D_{i} \tau\right. \\
& \left.+2^{(5)} J_{i}\left(\tau_{j}^{i}-\frac{1}{3} \delta_{j}^{i} \tau\right)\right] .
\end{aligned}
$$

From this equation it follows that the divergence of the corrections from the bulk space to the effective field equations is completely determined by the distribution of matter on the brane, though the total behaviour of these quantities must obey the evolution equation in the bulk. In the case of a vacuum on the brane $\left(\tau_{j}^{i}=0\right)$, equations (66), (72) and (70) are reduced to the form

$$
\begin{gathered}
G_{i j}=-\Lambda h_{i j}-W_{i j}-3 \kappa_{5}^{2} U_{i j}, \\
D^{i}\left(W_{i j}+3 \kappa_{5}^{2} U_{i j}-\frac{1}{2} \kappa_{5}^{2} h_{i j} P\right)=0,
\end{gathered}
$$

where equation (74) is of an integrability condition on the brane. In the case of empty bulk and vanishing cosmological constant $\Lambda=0$, one can solve equations (73) and (174) using a special metric anzatz on the brane that makes the system of equations closed [32]. Since 
the tensor $W_{i j}$ is traceless, the authors of paper [33] made a prescription for mapping fourdimensional general relativity solutions with traceless energy-momentum tensor to vacuum braneworld solutions in five-dimensional gravity. In particular, they found the exact solution for a static black hole on a 3-brane with a tidal charge arising due to gravitational influnce of the fifth dimension on the brane. A similar approach has been used in [34] to construct exact solutions for rotating and charged black holes localized on the brane. It is clear that with further assumptions on the metric structure on a 3-brane and on the projections $P$ and $U_{i j}$ of the bulk energy-momentum tensor, one can extend the link between some solutions in general relativity and braneworlds to include contributions from non-empty bulk space as well.

\section{THE EVOLUTION EQUATIONS}

As we have emphasized above, the effective gravitational field equations (58) and ([66) on the brane in general are not closed and the evolution equations into the bulk are needed to be solved for the projected bulk curvature and energy-momentum tensors. In this section we shall derive these evolution equations. First, we note that using equations (56) and (57) along with (51), for the traceless tensor $W_{i j}$ defined from the "electric part" of the bulk Riemann tensor we obtain

$$
\begin{aligned}
W_{i j}= & -\frac{1}{N}\left[\left(\partial_{5}-£_{\vec{N}}\right)\left(K_{i j}-\frac{1}{4} K h_{i j}\right)+D_{i} D_{j} N\right]-\frac{1}{2} K K_{i j}+K_{i}^{m} K_{m j} \\
& +\frac{1}{4} h_{i j}\left(K_{l m} K^{l m}+\frac{1}{N} \square N\right) .
\end{aligned}
$$

We recall that the "magnetic part", $B_{j i m}$, of the Riemann tensor defined in equation (50) is not traceless. It may be useful to construct from this quantity its traceless counterpart. This is given by

$$
M_{j i m}=B_{j i m}+\frac{2}{3} \kappa_{5}^{2} h_{j[i} J_{m]},
$$

where we have used equation (43). It turns out that this quantity is exactly the same as the "magnetic part" of the Weyl tensor introduced in [22]. We now need to establish the equations governing the evolution of the quantities $W_{i j}$ and $M_{j i m}$ into the bulk. For this purpose, it is convenient to start with equation (55), which can also be rewritten in the form

$$
R_{i j}=\frac{1}{2} h_{i j}\left(\Lambda_{5}-\kappa_{5}^{2} P\right)-W_{i j}-3 \kappa_{5}^{2} U_{i j}-K_{i}^{m} K_{m j}+K K_{i j}
$$


Acting on both sides of this equation by the operator $\partial_{5}-£_{\vec{N}}$ one can express the evolution of $W_{i j}$ in terms of the evolution of the braneworld Ricci tensor $R_{i j}$ which, in turn, can be determined through the five-dimensional Bianchi identities

$$
\nabla_{[A}{ }^{(5)} R_{B C] D E}=0 .
$$

Using equations (30) and (38) along with (49) one can decompose (78) to obtain the following set of identities

$$
\begin{gathered}
D_{[i} R_{j l] m s}=0 \\
D_{[m} B_{|j| l k]}+K_{[m}^{i} R_{k l] i j}=0, \\
\frac{1}{N}\left(\partial_{5}-£_{\vec{N}}\right) B_{j m l}-2\left(D_{[m} A_{l] j}+K_{[m}^{i} B_{l] j i}-A_{j[l} a_{m]}\right) \\
-K_{j}^{i} B_{i m l}-{ }^{(5)} R_{i j m l} a^{i}=0, \\
\frac{1}{2 N}\left(\partial_{5}-£_{\vec{N}}\right) R_{i j l m}+D_{[l} B_{m] j i}+a_{[i} B_{j] l m}+a_{[l} B_{m] i j}-2 K_{[i \mid l l} D_{m]]} a_{j]} \\
+R_{l m s[i} K_{j]}^{s}-2 a_{[i} K_{j][l} a_{m]}=0,
\end{gathered}
$$

here and in what follows the vertical bars at both sides of an index, or indices indicate that the indices are omitted from (anti)-symmetrization.

Taking the trace of equation (82) and comparing the result with equation (777) after acting on it by the operator $\partial_{5}-£_{\vec{N}}$, we obtain the following evolution equation for the projected bulk curvature and energy-momentum tensors involved in the effecive field equations on the brane

$$
\begin{aligned}
\frac{1}{N}\left(\partial_{5}-£_{\vec{N}}\right)( & \left.W_{i j}+3 \kappa_{5}^{2} U_{i j}+\frac{1}{2} \kappa_{5}^{2} P h_{i j}\right)=\left(D^{m}-2 a^{m}\right) M_{(i j) m}+3\left(K_{i}^{m} W_{j) m}+\kappa_{5}^{2} K_{(i}^{m} U_{j) m}\right) \\
& -\left[K W_{i j}+\left(K_{i j} K_{l m}-K_{i m} K_{j l}\right) K^{l m}-R_{l i m j} K^{l m}-\frac{1}{6} \Lambda_{5}\left(K_{i j}-h_{i j} K\right)\right] \\
& +\frac{2}{3} \kappa_{5}^{2}\left[2\left(J_{(i} a_{j)}+\frac{1}{2} h_{i j} J_{m} a^{m}\right)-\left(D_{(i} J_{j)}+\frac{1}{2} h_{i j} D_{m} J^{m}\right)\right] \\
& +\frac{1}{4} \kappa_{5}^{2}\left[\frac{2}{3}{ }^{(5)} T\left(K_{i j}+\frac{1}{2} h_{i j} K\right)-P h_{i j} K\right]
\end{aligned}
$$

In obtaining this equation we have also used Bianchi identities given by equations (779) and (801). Taking into account the expression (76) in equation (81) we we arrive at the evolution equation for the traceless "magnetic" part of the Weyl tensor 


$$
\begin{aligned}
\frac{1}{N}\left(\partial_{5}-£_{\vec{N}}\right) M_{j m l} & =2\left(D_{[m} W_{l] j}+K_{[m}^{i} M_{l] j i}+W_{j[m} a_{l]}\right)+K_{j}^{i} M_{i m l}+a^{i(5)} R_{i j m l} \\
& -\frac{2}{3} \kappa_{5}^{2}\left[\frac{1}{N} h_{j[l}\left(\partial_{5}-£_{\vec{N}}\right) J_{m]}+2\left(K_{[l}^{i} h_{m][i} J_{j]}+K_{j[l} J_{m]}\right)+K_{j}^{i} h_{i[m} J_{l]}\right] \\
& +\frac{1}{2} \kappa_{5}^{2} h_{j[l} D_{m]}\left(P-\frac{1}{3}{ }^{(5)} T\right)-\frac{1}{2}\left[\kappa_{5}^{2}\left(P-\frac{1}{3}{ }^{(5)} T\right)+\frac{2}{3} \Lambda_{5}\right] h_{j[l} a_{m]}(84)
\end{aligned}
$$

The equations (75), (83) and (84) generalize the corresponding equations obtained in [22] to the case of non-geodesic ADM type slicing of the bulk spacetime with nonvanishing matter fields. We note that here we deal with the tensor $W_{i j}$ which looks more simpler and it coincides with $E_{i j}$ when the bulk is empty. We see that the evolution equations are significantly modified due to the nonvanishing acceleration of spacelike normals to the brane surface, as well as the presence of the other fields in the bulk. Clearly, these equations are needed to be solved with appropriate boundary conditions on the brane. They are given by equation (172) and also by

$$
\left[M_{j m l}\right]=-2 \kappa_{5}^{2} D_{[l}\left(\tau_{m] j}-\frac{1}{3} h_{m] j} \tau\right)-\frac{2}{3} \kappa_{5}^{2} D_{i}\left(h_{l[j} \tau_{m]}^{i}\right),
$$

which follows from the Israel junction conditions (45) along with taking into account equation (431). In the case of a vacuum in the bulk ${ }^{(5)} T_{A B} \equiv 0$, the above equations are simplified to have the form

$$
\begin{gathered}
\frac{1}{N}\left(\partial_{5}-£_{\vec{N}}\right) W_{i j}=\left(D^{m}-2 a^{m}\right) M_{(i j) m}+3 K_{(i}^{m} W_{j) m}-K W_{i j}+R_{l i m j} K^{l m} \\
+\left(K_{i m} K_{j l}-K_{i j} K_{l m}\right) K^{l m}+\frac{1}{6} \Lambda_{5}\left(K_{i j}-h_{i j} K\right), \\
\frac{1}{N}\left(\partial_{5}-£_{\vec{N}}\right) M_{j m l}=2\left(D_{[m} W_{l] j}+K_{[m}^{i} M_{l] j i}+W_{j[m} a_{l]}\right)+K_{j}^{i} M_{i m l} \\
+a^{i(5)} R_{i j m l}-\frac{1}{3} \Lambda_{5} h_{j[l} a_{m]} .
\end{gathered}
$$

When passing to the Gausssian normal coordinates these equations are in agreement with those obtained in [22]. Thus, the equations governing the evolution of quantities $W_{i j}$ and $M_{j m l}$ into the fifth dimension are the desired supplements to the effective field equations on the 3-brane given in (42), (43) and (66) making the full system of equations closed. 


\section{CONCLUSION}

We have generalized the effective gravitational field equations on and off a 3-brane with $Z_{2}$ symmetry to the case where there exists an arbitrary energy-momentum tensor in the bulk. We have adopted a general coordinate setting introducing the lapse scalar field and the shift vector field, in accordance with their corresponding counterparts in ADM type slicing of a spacetime in four-dimensional general relativity. The use of the ADM type coordinates provides complete freedom in the slicing of a five dimensional bulk spacetime by pushing the timelike hypersurfaces forward in the fifth dimension at arbitrary rates in different positions. We have shown that the form of the on-brane effective gravitational field equations obtained earlier by Shiromizu, Maeda and Sasaki in the framework of the Gaussian normal coordinates continues to be held in the ADM type coordinate setting, however, the evolution equations into the bulk are significantly changed due to both the acceleration of normals inherent in the ADM approach and the presence of other bulk fields. We have also shown that the gravitational effects of the fifth dimension on a $Z_{2}$-symmetric 3 -brane may be described by means of a traceless second-rank tensor, properly constructed from the "electric" part of the Riemann tensor from the bulk space.

It should be emphasized that the formalism used in this paper might be very useful in studying gravitational perturbations in braneworld gravity and cosmology. The use of a general lapse function and shift vector may provide one with a new gauge, thereby taking into account the "brane -bending" type effects consistently. In particular, it would be interesting to explore the effects of the universal aspects of linearized gravity in a 3-braneworld, solving the on-brane and the off-brane equations for corresponding metric fluctuations.

\section{ACKNOWLEDGMENTS}

We would like to thank R. Maartens for discussions and valuable comments. One of us (A.N.) also thanks M. Sasaki for useful discussions at an early stage of this work.

[1] J. Polchinski, TASI Lectures on D-branes, hep-th/9611050

[2] P. Horava and E. Witten, Nucl. Phys. B 460, 506 (1996). 
[3] E. Witten, Nucl. Phys. B 471, 135 (1996).

[4] A. Lukas, B.A. Ovrut, K.S. Stelle, and D. Waldram, Phys. Rev. D 59, 086001 (1999).

[5] V. A. Rubakov and M. E. Shaposhnikov, Phys. Lett. B 125, 136 (1983).

[6] K. Akama, Lecture Notes in Physics, 176, 267 (1982) hep-ph/0001113

[7] M. Visser, Phys. Lett. B 159, 22 (1985).

[8] G. W. Gibbons and D. L. Wiltshire, Nucl. Phys. B 287, 717 (1987).

[9] N. Arkani-Hamed, S. Dimopoulos, and G. Dvali, Phys. Lett. B 429, 263 (1998); I. Antoniadis, N. Arkani-Hamed, S. Dimopoulos, and G. Dvali, Phys. Lett. B 436, 257 (1998).

[10] R. Emparan, M. Masip, and R. Rattazzi, Phys. Rev. D 65, 064023 (2002)

[11] S. B. Giddings and S. Thomas, Phys. Rev. D 65, 056010 (2002).

[12] S. Dimopoulos and G. Landsberg, Phys. Rev. Lett. 87, 161602 (2001).

[13] L. Randall and R. Sundrum, Phys. Rev. Lett. 83, 3370 (1999).

[14] L. Randall and R. Sundrum, Phys. Rev. Lett. 83, 4960 (1999).

[15] C. Csaki, TASI Lectures on Extra Dimensionsand Branes hep-ph/0308112.

[16] R. Maartens, Brane-World Gravity gr-qc/0312059.

[17] G. Gabadadze, ICTP Lectures on Large Extra Dimensions hep-ph/0308112

[18] V. Rubakov, Phys. Usp. 44, 871 (2001); Usp. Fiz. Nauk 171, 913 (2001).

[19] J. Garrigia and T. Tanaka, Phys. Rev. Lett. 84, 2778 (2000).

[20] S. B. Giddings, E. Katz and L. Randall, J. High Energy Phys. 0003, 23 (2000).

[21] A. Chamblin and G.W. Gibbons, Phys. Rev. Lett. 84, 1090, (2000)

[22] T. Shiromizu, K. Maeda, and M. Sasaki, Phys. Rev. D 62, 024012 (2000).

[23] M. Sasaki, T. Shiromizu and K. Maeda, Phys. Rev. D 62, 024008 (2000).

[24] K. Maeda and D. Wands, Phys. Rev. D 62, 124009 (2000).

[25] K. Maeda and T. Torii, Phys. Rev. D 69, 024002 (2004).

[26] C. W. Misner, K. S. Thorne, and J. A. Wheeler, Gravitation, W. H. Freeman and Company (1973).

[27] R. Arnowitt, S. Deser, and C.W. Misner, In Gravitation: an introduction to current research, L. Witten, ed. Wiley, New York, p.227 (1962); gr-qc/0405109

[28] G.W. Gibbons and S.W. Hawking, Phys. Rev. D 12, 2752 (1977).

[29] W. Israel, Nuovo Cimento 44B, 1 (1966); Errata-ibid 48B, 463 (1967).

[30] E. Poisson, A Relativist's Toolkit: The Mathematics of Black Hole Mechanics, Cambridge 
University Press (2004)

[31] L.D. Landau and E.M. Lifshitz, The Classical Theory of Fields, Pergamon Press (1975).

[32] R. Maartens, Phys. Rev. D 62, 084023 (2000).

[33] N. Dadhich, R. Maartens, P. Papadopoulos, and V. Rezania, Phys. Lett. B 487, 1 (2000).

[34] A.N. Aliev and A.E. Gumrukcuoglu, in preparation (2004) 